\title{
IMPACTO DE LA ACTIVACIÓN EMOCIONAL EN EL RECONOCIMIENTO DE EMOCIONES EN PERSONAS ADULTAS CON TRASTORNOS DEL ESPECTRO DEL AUTISMO Y DISCAPACIDAD INTELECTUAL
}

\author{
IMPACT OF EMOTIONAL ACTIVATION ON \\ THE RECOGNITION OF EMOTIONS IN \\ ADULTS WITH AUTISM SPECTRUM \\ DISORDERS AND INTELLECTUAL \\ DISABILITY
}

\section{MARINA JODRA $^{1}$ Y DOMINGO GARCÍA-VILLAMISAR $^{1}$}

Cómo referenciar este artículo/How to reference this article:

Jodra, M. y García-Villamisar, D. (2019). Impacto de la activación emocional en el reconocimiento de emociones en personas adultas con Trastornos del Espectro del Autismo y Discapacidad Intelectual [ Impact of Emotional Activation on the Recognition of Emotions in Adults with Autism Spectrum Disorders and Intellectual Disability]. Acción Psicológica, 16(2), 103-118. https://doi.org/10.5944/ap.16.2.25566

\section{Resumen}

Uno de los síntomas nucleares de los Trastornos del Espectro del Autismo (TEA) es el déficit en el área socioemocional, mostrando patrones de respuesta atípicos ante los estímulos sociales y respondiendo con distintos niveles de activación o arousal (hiperexcitación o hipoexcitación). Algunas investigaciones han podido comprobar como la exposición a imágenes de valencia

Correspondence address [Dirección para correspondencia]: Marina Jodra Chuan. Universidad Complutense de Madrid, España.

Email: majodra@ucm.es

ORCID: Marina Jodra (http://orcid.org/0000-0002-2467-7097) y Domingo García-Villamisar (https://orcid.org/00000001-5526-6496).

${ }^{1}$ Universidad Complutense de Madrid, España.

Recibido: 12 de agosto de 2019.

Aceptado14 de noviembre de 2019. negativa tiene efectos en el reconocimiento posterior de emociones en población sin TEA. En cambio, los escasos estudios que existen acerca de la permeabilidad emocional en personas con TEA, muestran patrones atípicos de respuesta fisiológica ante la inducción de emociones. En la presente investigación se analiza la repercusión de la inducción emocional «negativa», a través de imágenes del International Affective Picture System (IAPS; Lang, Bradley y Cuthbert, 1995), en el reconocimiento de emociones a través del rostro mediante la Facial Discrimination Battery (FDB; Rojahn, Esbensen y Hoch, 
2006), en un grupo de 31 personas adultas con TEA y discapacidad intelectual. Se realizó una ANOVA de medidas repetidas y los resultados demostraron la existencia de cierta «impermeabilidad» emocional, ya que la inducción de emociones no influyó en el reconocimiento emocional posterior. Estos resultados son coherentes con el modelo de hipoexcitación, que defiende que las personas con TEA experimentan menos excitación o recompensa cuando atienden a los estímulos sociales.

Palabras clave: Autismo; Comportamiento social; Desarrollo emocional; Emociones; Respuesta emocional; Trastornos del espectro del autismo.

\section{Abstract}

One of the core symptoms of Autism Spectrum Disorders (ASD) is the deficit in the socio-emotional area, showing atypical response patterns to social stimuli and responding with different levels of activation or arousal (hyperexcitation or hypoexcitation). Some investigations have shown how exposure to negative valence images has an effect on the subsequent recognition of emotions in a population without ASD. In contrast, the few studies that exist on emotional permeability in people with ASD show atypical patterns of physiological response to the induction of emotions. In the present investigation, the impact of "negative" emotional induction is analyzed, through images of the International Affective Picture System (IAPS, Lang et al., 1995), in the recognition of emotions through the face through Facial Discrimination Battery (FDB; Rojahn et al., 2006), in a group of 31 adults with ASD and intellectual disability. An ANOVA of repeated measures was performed and the results demonstrated the existence of a certain emotional "impermeability", since the induction of emotions did not influence the subsequent emotional recognition. These results are consistent with the hypoexcitation model, which argues that people with ASD experience less excitement or reward when they attend to social stimuli.

Keywords: Autism; Social behavior; Emotional development; Emotions; Emotional response; Autism spectrum disorders.

\section{Introducción}

Actualmente el déficit en el área socio-emocional es uno de los criterios necesarios para diagnosticar un Trastorno del Espectro del Autismo (TEA), siendo el primer bloque sintomático junto a las alteraciones en la comunicación en el DSM 5 (DSM-5; APA, 2013).

Diversos estudios han demostrado la existencia de un déficit específico en el reconocimiento y comprensión de emociones en personas con autismo (Blain, Peterman y Park, 2017; García-Villamisar y Polaino-Lorente, 2000; García-Villamisar, Rojahn, Zaja y Jodra, 2010; Uljarevic y Hamilton, 2013). Algunas investigaciones limitan el déficit al reconocimiento de emociones muy concretas tales como el miedo (De Jong, van Engelund y Kemner, 2008; Pelphrey et al., 2002), la tristeza (Boraston, Blakemore, Chilvers y Skuse, 2007), las emociones de «valencia positiva» (Herpers et al., 2019) o las emociones «negativas» (Ashwin, Chapman, Colle y Baron-Cohen, 2006; Humphreys, Minshew, Leonard y Behrmann, 2007; Ioannou et al., 2017), mostrando en líneas generales menos atención a las mismas (Baron-Cohen et al., 2000). También se ha observado cierta falta de interés por parte de las personas con autismo hacia las emociones de los demás (Begeer et al., 2006; Pelphrey et al., 2002; Weeks y Hobson, 1987), y una atención deficitaria ante estímulos sociales (Chevallier, Huguet, Happé, George y Conty, 2013; Mathersul, McDonald y Rushby, 2013a). Otras investigaciones han observado un patrón diferente de atención ante estímulos emocionales, comprobando en personas con TEA una mayor atención a estímulos amenazantes (Ghosn et al., 2019). Además, se ha detectado menos expresividad en las interacciones sociales cuando se les compara con personas con desarrollo típico o discapacidad intelectual sin TEA (Czapinski y Bryson, 2003; Kasari, Sigman, Mundy y Yirmiya, 1990; Stagg, Slavny, Hand, Cardoso y Smith, 2014; Yirmiya, Kasari, Sigman y Mundy, 1989). En personas con discapacidad intelectual y autismo se detectó menos activación ante la dirección de la mirada de otra persona (Kylliäinen y Hietanen, 2006) o ante situaciones de peligro (Corona, Dissanayake, Arbelle, Wellington y Sigman, 1998). Algunas investigaciones resaltan la existencia de dificultades significativas cuando la percepción emocional se hace a través de rostros (Walsh, Crei- 
ghton y Rutherford, 2016; Harms, Martin y Wallace, 2010).

Una de las teorías que ha intentado explicar estos patrones de respuesta atípicos en el TEA defiende que ante los estímulos sociales las personas con autismo responden con distintos niveles de activación o arousal (Kaartinen, Puura, Himanen, Nevalainen y Hietanen, 2016; Kylliäinen et al., 2012; Riby, Whittle y Doherty-Sneddon, 2012). El modelo de hiperexcitación sugiere que el sistema nervioso autónomo de los individuos con TEA es «elevado», están constantemente en una alerta máxima (Bal et al., 2010; Hirstein, Iversen y Ramachandran, 2001). En cambio, el modelo de hipoexcitación, defiende que las personas con TEA experimentar menos excitación o recompensa cuando atienden a los estímulos sociales (Mathersul, McDonald y Rushby, 2013b).

Los estudios realizados con el IAPS (International Affective Picture System; Lang et al., 1995), demostraron que la inducción de emociones influye tanto en variables fisiológicas como la respuesta galvánica o tasa cardiaca (Choi et al., 2017; Lang, Greenwald, Bradley y Hamm, 1993, Zangróniz, Martínez-Rodrigo, Pastor, López y Fernández-Caballero, 2017), como en estructuras cerebrales como la amígdala, el hipocampo, el cortex frontal ventromedial y el cortex visual (Britton, Taylor, Sudheimer y Liberzon, 2006), activando distintas zonas dependiendo de si las imágenes son de valencia positiva o negativa (Nielen et al., 2009).

Algunas investigaciones han podido comprobar como la exposición a imágenes de valencia negativa tiene efectos en el reconocimiento posterior de emociones. Se ha observado un reconocimiento más rápido cuando las imágenes son negativas o positivas (Gordillo et al., 2010; Redondo y Fernández-Rey, 2010), un efecto positivo en el rendimiento en tareas de memoria de trabajo después de visualizar emociones «negativas» a través del rostro (Schechtman Belham et al., 2017; Machinskaya, Rozovskaya, Kurgansky y Pechenkova, 2016), o un mejor reconocimiento de la «sorpresa» y un déficit en cuanto al «disgusto» en sujetos expuestos a un estímulo que provocan ansiedad social (Daudelin-Peltier, Forget, Blais, Deschênes y Fiset, 2017).
Existe cierta relación entre la sintomatología ansiosa y depresiva (humor negativo) y la predisposición a reconocer los estímulos como negativos (Dowens y Calvo, 2003; Powell y Hemsley, 1984), un rendimiento menor en tareas de reconocimiento (Surguladze et., 2004) o, por otro lado, una insensibilidad ante las emociones (Rottenberg, Joormann, Brozovich y Gotlib, 2005). Estos estudios también se han llevado a cabo con personas sin depresión o ansiedad, a las que se induce humor positivo y negativo. Los resultados muestran una predisposición mayor de los sujetos con humor negativo por la identificación de los estímulos como negativos (Bouhuys, Bloem y Groothuis, 1995; Niedenthal, Halberstadt y Margolin, 2000; Schmidt y Mast, 2010; Yi, Murry y Gentzler, 2016) y un mayor rendimiento en este tipo de tareas en las personas con humor positivo (Ovsyannikova, 2016). También han relacionado estas variables con la edad, comprobando una predisposición mayor a percibir estímulos negativos cuando se inducen emociones negativas y la persona tiene mayor edad (Lawrie, Jackson y Phillips, 2018).

En el caso de personas con TEA, existen pocos estudios acerca de la inducción o permeabilidad emocional. Aunque sí se ha observado una reacción fisiológica alterada ante la inducción emocional con el IAPS, a través de la presión arterial y la tasa cardíaca (Bölte, FeineisMatthews y Poustka, 2008; Kaartinen et al., 2012; Kylliäinen et al., 2012; Mathersul et al., 2013b). Stagg, Davis y Heaton (2013) detectaron una relación entre la baja respuesta galvánica ante estímulos sociales, como la dirección de la mirada, y el retraso en el desarrollo del lenguaje. Por el contrario, otros estudios no han podido comprobar estas diferencias (Louwerse et al., 2014).

En síntesis, los estudios revisados ponen de relieve que las personas con autismo tienen dificultades en el área socioemocional y estas pueden estar causadas por una hipoexcitación o hiperexcitación ante estímulos de este tipo. Los indicios acerca de la posible «impermeabilidad emocional» ante los estímulos que les rodean, podrían relacionarse con estas dificultades.

Por ello, el objetivo de esta investigación fue averiguar si las personas adultas con autismo y discapacidad intelectual se ven afectadas, ante la inducción de emociones de valencia negativa, en la realización de tareas de reconoci- 
miento de emociones a través del rostro. Se planteó la siguiente hipótesis: las personas con TEA serán impermeables a la inducción de emociones y, por lo tanto, no existirán diferencias significativas entre la primera (antes de inducir emociones negativas) y segunda aplicación de las tareas de reconocimiento emocional (después de la inducción emocional)..

\section{Método}

\section{Participantes}

La muestra seleccionada para realizar la presente investigación consta de 31 personas adultas con discapacidad intelectual y TEA, de los cuales 19 son varones $(61.2 \%)$ y 12 mujeres $(38.7 \%)$. Los participantes han sido seleccionados en una institución sin ánimo de lucro dedicada a la atención de personas adultas con autismo de la Comunidad de Madrid. Todos han sido diagnosticados por especialistas en la evaluación de Trastornos del Espectro del Autismo, según los criterios diagnósticos de la Asociación Americana de Psiquiatría (DSM-IV; APA, 1994).

El único criterio de exclusión fue que ningún participante se estuviera medicando con fármacos que pudiesen perjudicar al desarrollo de tareas cognitivas (tratamientos con psicotrópicos o corticoesteroides). La selección de la muestra se realizó contando con todas las personas que cumplían con este criterio en la asociación donde se acu 1 . dió, no resultando excluido ninguno de los usuarios de la entidad.

Los datos demográficos se pueden observar en la Tabla 1, donde se aprecian las medias correspondientes a la edad mental y las puntuaciones en distintas áreas del desarrollo (socialización, habilidades de la vida diaria y comunica 2 ción), medidas a través del Vineland Adaptive Behavior Scales (VABS; Sparrow, Balla y Cicchetti, 1984). Igualmente se incluye la distribución en función del sexo, expresada en porcentajes.
Tabla 1

Características demográficas de los participantes

\begin{tabular}{lrc}
\hline & \multicolumn{2}{c}{ TEA } \\
& $\mathbf{N}=\mathbf{3 1}$ \\
\hline & 35.76 & \multicolumn{1}{c}{$\mathbf{D T}$} \\
\hline Edad cronológica & 4.74 & 3.33 \\
Edad mental no verbal & & \\
VABS & 39.03 & 25.32 \\
Socialización & 51.03 & 29.11 \\
Comunicación & 70.70 & 29.11 \\
Habilidades de la vida diaria & $\mathbf{( \% )}$ & \\
Sexo & 61.2 & \\
Varones & 38.7 & \\
Mujeres &
\end{tabular}

Nota: VABS = Vineland Adaptive Behavior Scales (Sparrow, Balla y Cicchetti, 1984).

\section{Instrumentos de evaluación}

\section{Variable dependiente: reconocimiento de emociones a través del rostro}

El reconocimiento de emociones a través del rostro se evaluó a través de la batería Facial Discrimination Battery (FDB; Rojahn et al., 2006; García-Villamisar et al., 2010). Consta de cuatro pruebas emocionales y dos pruebas no emocionales. Se contabilizaron las respuestas correctas e incorrectas de los participantes y se calculó el porcentaje de respuestas correctas para cada subtest. Las tareas emocionales son las siguientes:

Tarea de Discriminación Facial (emocional). Compuesta por 36 ítems: 12 de tristeza, 12 de felicidad y 12 con expresiones faciales neutrales. En cada diapositiva aparece un rostro y se pregunta a los participantes qué emoción expresa. Primero se muestran unos ítems de práctica para valorar si la persona es capaz de seguir las instrucciones y realizar el test.

Tarea de Reconocimiento Facial. Esta tarea contiene 21 ítems, cada uno de ellos compuesto por cinco rostros. El nombre de una emoción aparece en cada diapositiva (feliz, neutral o triste) y se tiene que identificar el rostro que mejor la representa (Figura 1).

3. Tarea de Identificación. Esta tarea contiene 20 ítems con un breve enunciado que narra lo que le ha ocurrido al protagonista y tres fotos que representan distintas emociones 


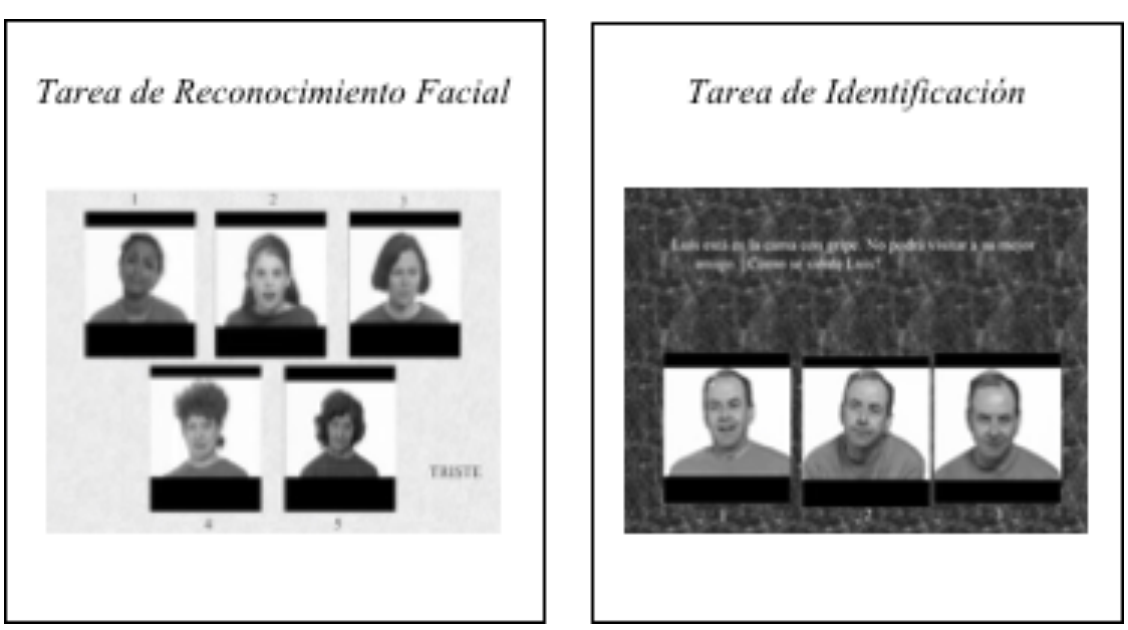

Figura 1. Diapositivas de la Batería de Emociones de Rojahn (García-Villamisar et al., 2010)

de la misma persona. Se pregunta qué imagen representa mejor lo que le ha ocurrido al protagonista (Figura 1).

Emparejar con la Muestra (Tarea Emocional). Esta tarea consta de 20 ítems con un rostro de muestra que se localiza centrado en la parte superior de la pantalla, y otros cinco rostros en la parte inferior de la diapositiva. Se pregunta al participante; "Mira a esta persona. ¿Cómo se siente? (imagen superior). Ahora mira a estas cinco personas de abajo y señala a la persona que se siente igual que la de arriba". Primero se enseñan unos ítems de práctica y después comienza el test.

La batería también consta de dos pruebas control que no tienen contenido emocional:

1. Tarea de Discriminación Facial según la Edad. Compuesta por 24 rostros de distintas edades ( 9 jóvenes, 10 de mediana edad y cinco de la $3^{\circ}$ edad), 12 mujeres y 12 hombres. En cada ítem aparece un solo rostro y se debe clasificar según una de las tres franjas de edad. Después de visualizar los primeros ítems de práctica, comienza el test.

2. Emparejar con la Muestra (Tarea de Identidad). Esta prueba esta compuesta por 10 diapositivas con el mismo formato que las de la Tarea Emocional de Emparejar con la Muestra. Se pide que se encuentre el rostro de la misma persona que la imagen de mues-
tra.Cinco ítems tienen a actores masculinos y cinco a actrices femeninas.

\section{Variable independiente: inducción de emociones negativas}

El International Affective Picture System (IAPS; Lang et al., 1995) es una batería con 900 diapositivas en color clasificadas en diferentes categorías emocionales. Forma parte de una colección de estímulos emocionales, junto con el ANEW (Affective Lexicon of English Words) y el IADS (International Affective Digitized Sound System), desarrollada en la Universidad de Florida por el NIMH Center for Emotion and Attention (CSEA).

El IAPS clasifica sus imágenes en tres dimensiones emocionales: agradable / desagradable; calma / excitación (arousal) y descontrol / control.

\section{Procedimiento}

El proceso de evaluación se inició tras haber obtenido consentimiento informado de todos los tutores legales de los participantes en este estudio. Todas las tareas se aplicaron de manera individual y con un único profesional como responsable. La tarea de inducción emocional ha re- 
querido la utilización de un ordenador y una pizarra digital, donde se les mostraban a los participantes las imágenes para la posterior realización de las pruebas.

Las imágenes del IAPS utilizadas en este estudio se seleccionaron después de consultar las medias y desviaciones típicas en la población general (Lang et al., 2005) y compartir el método de selección de las mismas con los autores de la batería.

Como imágenes «agradables» se escogieron aquellas que tienen una media entre 8 y 9 , «desagradables» entre 1 y 2 , «excitantes» entre 7 y 9 , «calmantes» entre 1 y 3 , que producen «descontrol» entre 1 y 3 y como imágenes que producen «control» las que tienen una media entre 7 y 9 . Por último, las imágenes que tienen una media entre 4.5 y 5.5 en las tres dimensiones emocionales fueron escogidas como imágenes neutras.

El objetivo principal de esta investigación fue comprobar si las personas con TEA experimentan desagrado, excitación y descontrol ante el visionado de estas imágenes. Para ello, se dividieron aleatoriamente en dos subgrupos, a uno de ellos se le presentaron 40 imágenes «neutras» y al otro 33 imágenes con contenido emocional.

La Batería de Emociones de Rojahn se aplicó a ambos grupos antes y después de la inducción experimental.

\section{Análisis estadístico}

El análisis estadístico llevado a cabo fue un ANOVA de medidas repetidas, mediante un modelo de dos factores con medidas repetidas de un factor. La variable dependiente fue el rendimiento emocional antes y después de la inducción y la variable intersujetos fue la inducción de emociones (negativas o neutras). Todos estos análisis se realizaron mediante el programa IBM SPSS Statistics 22 para Windows.

\section{Resultados}

En la Tabla 2 se pueden observar los resultados del ANOVA de medidas repetidas. Los contrastes intrasujetos revelan como el tiempo de medida (primera o segunda aplicación de las tareas), influye significativamente en el rendimiento emocional $\left(\mathrm{F}(7,15)=3.60, p=.01, \mathrm{\eta}^{2}=.62\right)$.

Por el contrario, ni la inducción de emociones $\left(\mathrm{F}(7,15)=1.42, p=.26, \mathrm{\eta}^{2}=.39\right)$ ni la interacción (momento de medida*inducción emocional) $(\mathrm{F}(7,15)=.75$, $\left.p=.63, \eta^{2}=.26\right)$, tuvieron una influencia significativa en el rendimiento en las tareas emocionales. Por lo tanto, las diferencias significativas entre la primera y la segunda aplicación no se pueden explicar por la inducción de emociones.

Tabla 2

Resultados del análisis de medidas repetidas para los efectos inter-sujetos (inducción o no inducción emocional) en el rendimiento en tareas de la Batería de emociones de Rojahn, antes y después de la inducción emocional

\begin{tabular}{|c|c|c|c|c|c|c|c|}
\hline & $1^{\circ}$ aplicación & & ción & & & & \\
\hline & $\mathbf{M}$ & DT & $\mathbf{M}$ & DT & $\mathbf{F}$ & $n^{2}$ & sig. \\
\hline R01 & 8.43 & 4.77 & 8.45 & 4.13 & $F_{(1,21)}=.01$ & .00 & .92 \\
\hline RO2 & 11.04 & 3.98 & 7.43 & 1.75 & $F_{(1,21)}=.47$ & .02 & .49 \\
\hline RO3 & 17.56 & 6.76 & 15.38 & 7.48 & $F_{(1,21)}=.18$ & .00 & .67 \\
\hline RO4 & 7.82 & 3.45 & 7.55 & 4.09 & $\mathrm{~F}_{(1,21)}=.51$ & .02 & .48 \\
\hline RO5 & 5.91 & 2.50 & 3.71 & 1.64 & $F_{(1,21)}=.05$ & .00 & .81 \\
\hline RO6 & 6.13 & 4.13 & 4.00 & 2.23 & $F_{(1,21)}=1.42$ & .06 & .24 \\
\hline
\end{tabular}

Nota: RO1= Tarea de Reconocimiento de la Batería de Emociones de Rojahn; RO2= Tarea de Identificación de la Batería de Emociones de Rojahn; RO3= Tarea de Discriminación Facial Emocional de la Batería de Emociones de Rojahn; RO4= Tarea de Discriminación Facial según la Edad de la Batería de Emociones de Rojahn; RO5= Tarea de Identidad de Emparejamiento a la Muestra de la Batería de Emociones de Rojahn; RO6= Tarea Emocional de Emparejamiento a la Muestra de la Batería de Emociones de Rojahn. 
En cuanto a la inducción o no de emociones neutras, el efecto del factor intersujetos no es significativo para ninguna de las tareas del estudio: la tarea de reconocimiento $\left(\mathrm{F}(1,21)=.01, p=.92, \mathrm{n}^{2}=.00\right)$, la tarea de identificación $\left(\mathrm{F}(1,21)=.47, p=.49, \mathrm{n}^{2}=.02\right)$, la tarea de discriminación facial emocional $\left(\mathrm{F}(1,21)=.18, p=.67, \mathrm{\eta}^{2}=.00\right)$, la tarea de discriminación facial según la edad $(\mathrm{F}(1,21)=$ $\left..51 ; p=.48, \eta^{2}=.02\right)$, la tarea de identidad de emparejamiento a la muestra $\left(\mathrm{F}(1,21)=.05, p=.81, \mathrm{n}^{2}=.00\right)$ y la tarea emocional de emparejamiento a la muestra de la Batería de Emociones de Rojahn $(\mathrm{F}(1,21)=1.42, p=.24$, $\left.\eta^{2}=.06\right)$. Además, el tamaño del efecto está por debajo de $.10\left(\eta^{2}\right)$ en todas las medidas.

\section{Discusión}

A través de los resultados obtenidos en este estudio se demuestra la existencia de cierta «impermeabilidad» emocional en personas adultas con autismo y discapacidad intelectual. El tiempo de medida (primera o segunda aplicación de las tareas) influye significativamente en el rendimiento emocional, en cambio la inducción de emociones no mostró una influencia significativa en el rendimiento en las tareas emocionales. Las diferencias significativas, entre la primera y la segunda aplicación de las tareas emocionales, no las puede explicar la inducción emocional. Por lo tanto, el rendimiento menor en la segunda aplicación puede deberse a otras variables como pérdida de atención o cansancio, pero no al proceso de inducción.

Estos resultados son contrarios a lo que se observa en población sin TEA, ya que cuando se visionan imágenes de valencia negativa se modifica el reconocimiento posterior de emociones (Daudelin-Peltier et al., 2017; Gordillo et al., 2010; Redondo y Fernández-Rey, 2010; Schechtman Belham et al., 2017). Además, al tratarse de población adulta se comprueba una diferencia más respecto a la población adulta sin TEA, ya que se ha detectado mayor predisposición para percibir estímulos negativos cuando se inducen emociones negativas y la persona tiene mayor edad (Lawrie et al., 2018).

El perfil socioemocional de las personas con TEA apunta a la existencia de cierta impermeabilidad a la hora de percibir y expresar emociones, tal como indican los resultados de esta investigación, que también son coherentes con el modelo de hipoexcitación que defiende que las personas con TEA experimentan menos excitación o recompensa cuando atienden a los estímulos sociales.

La literatura encontrada que puede preceder estos hallazgos, son estudios acerca de la capacidad de responder a las emociones de las personas con autismo. Entre estas investigaciones se encuentran aquellas que defienden la existencia de un déficit específico en el reconocimiento y comprensión de emociones (Ashwin et al., 2006; Blain et al., 2017; Boraston et al., 2007; De Jong et al., 2008; García-Villamisar y Polaino-Lorente, 2000; García-Villamisar et al., 2010; Humphreys et al., 2007; Ioannou et al., 2017; Pelphrey et al., 2002; Uljarevic y Hamilton, 2013). Este déficit está acompañado de cierta falta de interés por parte de las personas con autismo hacia las emociones de los demás (Begeer et al., 2007; Pelphrey et al., 2002; Weeks y Hobson, 1987), y una menor atención a estímulos sociales (Chevallier et al., 2013; Mathersul et al., 2013a). Además, las personas con TEA parecen menos expresivas en las interacciones sociales, mostrándose más neutrales que personas con discapacidad intelectual y desarrollo típico sin TEA (Czapinski y Bryson, 2003; Kasari et al., 1990; Stagg et al., 2014; Yirmiya el al., 1989). Sus respuestas suelen ser menos empáticas y se han detectado menos pulsaciones cuando responden a las emociones de otras personas (Corona et al., 1998). En personas con discapacidad intelectual y autismo se detectó menos excitación o atención ante situaciones de peligro (Corona et al., 1998) o la dirección de la mirada de otra persona (Kylliäinem y Hietanen, 2006).

Por último, se ha observado una reacción fisiológica alterada ante la inducción emocional con el IAPS, a través de la presión arterial y tasa cardiaca (Bölte et al., 2008; Kaartinen et al., 2012; Kylliäinen et al., 2012), o correlaciones significativas entre la respuesta galvánica ante estímulos sociales y el retraso en el desarrollo del lenguaje (Stagg et al., 2013). Estos hallazgos también se pueden analizar desde esta posible «impermeabilidad» emocional que produciría estos patrones atípicos de respuestas fisiológicas. 
Desde la perspectiva de las implicaciones prácticas, estos resultados muestran una cierta especificidad en la percepción de los estímulos emocionales en las personas con TEA. Se observa una «impermeabilidad» ante estímulos emocionales que se debería tener en cuenta a la hora de desarrollar intervenciones terapéuticas adaptadas a estas características.

\section{Limitaciones}

Los resultados del presente estudio apuntan a la existencia de cierta impermeabilidad emocional en personas adultas con TEA y discapacidad intelectual. En futuros estudios sería recomendable tener en cuenta las respuestas fisiológicas, como respuesta galvánica o tasa cardiaca, ya que complementaría la información acerca de la inducción emocional en esta población. También se debería tomar como población a personas con distintos perfiles, con y sin discapacidad intelectual.

Una de los síntomas principales de los TEA son las dificultades en el área de la comunicación y alguno de los participantes en este estudio carecía de lenguaje oral. Dentro de las tareas desarrolladas, alguna no estaba adaptada a esta característica y se ha intentado subsanar utilizando lengua de signos o pictogramas. Esta problemática ha podido ocasionar que algún resultado obtenido esté por debajo del desarrollo real de la persona, debido a una escasa adaptación de los instrumentos de evaluación existentes a las diversidades funcionales que se dan en los TEA.

Por último, el número de participantes puede restringir el poder para detectar relaciones causales entre variables. Esta limitación, aunque debe ser tenida en cuenta, queda atenuada si tenemos en cuenta que otros estudios han presentado un tamaño muestral igual o incluso inferior.

\section{Referencias}

American Psychiatric Association. (1994). Diagnostic and Statistical Manual of Mental Disorders (4 ed.). Washington, DC: Autor.
American Psychiatric Association. (2013). Diagnostic and Statistical Manual of Mental Disorders (5 ed.). Washington, DC: Autor.

Ashwin, C., Chapman, E., Colle, L. y Baron-Cohen, S. (2006). Impaired Recognition of Negative Basic Emotions in Autism: A Test of the Amygdala Theory. Social Neuroscience, 1, 349-363. https://doi.org/10.1080/17470910601040772

Bal, E., Harden, E., Lamb, D., Van Hecke, A. V., Denver, J. W. y Porges, S. W. (2010). Emotion Recognition in Children with Autism Spectrum Disorders: Relations to Eye Gaze and Autonomic State. Journal of Autism and Developmental Disorders, $\quad 40(3)$, 358-370. https://doi.org/10.1007/s10803-009-0884-3

Baron-Cohen, S., Ring, H. A., Bullmore, E. T., Wheelwright, S., Ashwin, C. y Williams, S. C. R. (2000). The Amygdala Theory of Autism. Neuroscience and Biobehavioral Reviews, 24, 355364. 7634(00)00011-7

Begeer, S., Meerum Terwogt, M., Rieffe, C., Stegge, H., Koot, H. M. y Blain, S. D. (2007). Do Children with Autism Acknowledge the Influence of Mood on Behaviour? Autism, 11(6), 503-521. https://doi.org/10.1177/1362361307083262

Blain, S. D., Peterman, J. S. y Park, S. (2017). Subtle Cues Missed: Impaired Perception of Emotion from Gait in Relation to Schizotypy and Autism Spectrum Traits. Schizophrenia Research, 183, 157-160. https://doi.org/10.1016/j.schres.2016.11.003

Bölte, S., Feineis-Matthews, S. y Poustka, F. (2008). Brief Report: Emotional Processing in HighFunctioning Autism. Physiological Reactivity and Affective Report. Journal of Autism and Developmental Disorders, 38(4), 776-781. https://doi.org/10.1007/s10803-007-0443-8 
Boraston, Z., Blakemore, S. J., Chilvers, R. y Skuse, D. (2007). Impaired Sadness Recognition is Linked to Social Interaction Deficit in Autism. Neuropsychologia, 45(7), 1501-1510. https://doi.org/10.1016/j.neuropsychologia.2006.1 1.010

Bouhuys, A. L., Bloem, G. M. y Groothuis, T. G. (1995). Induction of Depressed and Elated Mood by Music Influences the Perception of Facial Emotional Expressions in Healthy Subjects. Journal of Affective Disorders, 33, 215-226. https://doi.org/10.1016/0165-0327(94)00092-n

Britton, J. C,. Taylor, S. F., Sudheimer, K. D. y Liberzon, I. (2006). Facial Expressions and Complex IAPS Pictures: Common and Differential Networks. NeuroImage, $\quad 31, \quad 906-919$. https://doi.org/10.1016/j.neuroimage.2005.12.050

Chevallier, C., Huguet, P., Happé, F., George, N. y Conty, L. (2013) Salient Social Cues are Prioritized in Autism Spectrum Disorders despite Overall Decrease in Social Attention. Journal of Autism and Developmental Disorders, 43, 1642-1651. https://doi.org/10.1007/s10803-012-1710-x

Choi, K. H., Kim, J., Kwon, O. S., Kim, M. J., Ryu, Y. H. y Park, J. E. (2017). Is Heart Rate Variability (HRV) an Adequate Tool for Evaluating Human Emotions? - A Focus on the Use of the International Affective Picture System (IAPS). Psychiatry Research, 251, 192-196. https://doi.org/10.1016/j.psychres.2017.02.025

Corona, R., Dissanayake, C., Arbelle, S., Wellington, P. y Sigman, M. (1998). Is Affect Aversive to Young Children with Autism? Behavioral and Cardiac Responses to Experimenter Distress. Child Development, 69, 1494-1502.

Czapinski, P. y Bryson, S. E. (2003). Reduced Facial Muscle Movements in Autism: Evidence for Dysfunction in the Neuromuscular Pathway? Brain and Cognition, 51(2), 177-179. https://doi.org/10.1016/S0278-2626(02)00541-9,
Daudelin-Peltier, C., Forget, H., Blais, C., Deschênes, A. y Fiset, D. (2017). The effect of acute social stress on the recognition of facial expression of emotions. Scientific Reports, 7, 1036. https://doi.org/10.1038/s41598-017-01053-3

De Jong, M. C., van Engeland, H. y Kemner, C. (2008). Attentional Effects of Gaze Shifts are Influenced by Emotion and Spatial Frequency, but not in Autism. Journal of the American Academy of Child and Adolescent Psychiatry, 47(4), 443-454. https://doi.org/10.1093\%2Fscan\%2Fnst084

Dowens, M. G. y Calvo, G. C. (2003). Genuine Memory Bias versus Response Bias in Anxiety. Cognition and Emotion, 17, 843-857. https://doi.org/10.1080/02699930244000381

García-Villamisar, D. y Polaino-Lorente, A. (2000). El autismo y las emociones. Nuevos hallazgos experimentales [Autism and Emotions. New Experimental Findings]. Valencia, España: Promolibro.

García-Villamisar, D., Rojahn, J., Zaja, R. H. y Jodra, M. (2010). Facial Emotion Processing and Social Adaptation in Adults with and Without Autism Spectrum Disorder. Research in Autism Spectrum Disorders, $\quad 4$, 755-762. https://doi.org/10.1016/j.rasd.2010.01.016

Ghosn, F., Perea, M., Castelló, J., Vázquez, M. A., Yáñez, N., Marcos, I., Sahuquillo, R., Vento, M., García-Blanco, A. (2019). Attentional Patterns to Emotional Faces Versus Scenes in Children with Autism Spectrum Disorders. Journal of Autism and Developmental Disorders, 49, 1484-1492. https://doi.org/10.1007/s10803-018-3847-8

Gordillo, F., Arana J. M., Mestas, L., Salvador, J., Meilán, J. J. G. y Carro, J. (2010). Emotion and Recognition Memory: The Discrimination of Negative Information as an Adaptive Process. Psicothema, 22(4), 765-771. 
Harms, M. B., Martin, A. y Wallace, G. L. (2010). Facial Emotion Recognition in Autism Spectrum Disorders: A Review of Behavioral and Neuroimaging Studies. Neuropsychology Review, 20, 290-322. https://doi.org/10.1007/s11065-0109138-6

Herpers, P. C. M., Bakker-Huvenaars, M. J., Greven, C. U., Wiegers, E. C., Nijhof, K. S., Baanders, A. N., Buitelaar, J. K. y Rommelse, N. N. J. (2019). Emotional Valence Detection in Adolescents with Oppositional Defiant Disorder/Conduct Disorder or Autism Spectrum Disorder. European Child \& Adolescent Psychiatry, 28(7), 1011-1022. https://doi.org/10.1007/s00787-019-01282-z

Hirstein, W., Iversen, P. y Ramachandran, V. S. (2001). Autonomic Responses of Autistic Children to People and Objects. Proceeding: Biological Sciences, $\quad 268, \quad$ 1883-1888. https://doi.org/10.1098\%2Frspb.2001.1724

Humphreys, K., Minshew, N., Leonard, G. L. y Behrmann, M. (2007). A Fine-grained Analysis of Facial Expression Processing in High-Functioning Adults with Autism. Neuropsychologia, 45, 685695. https://doi.org/10.1016/j.neuropsychologia.2006.0 8.003

Ioannou, C., El Zein, M., Wyart, V., Amsellem, F., Delorme, R., Chevallier, C. y Grèzes, J. (2017). Shared Mechanism for Emotion Processing in Adolescents with and without Autism. Scientific Reports, 7. https://doi.org/10.1038\%2Fsrep42696

Kaartinen, M., Puura, K., Himanen, S. L., Nevalainen, J. y Hietanen, J. K. (2016). Autonomic Arousal Response Habituation to Social Stimuli Among Children with ASD. Journal of Autism and Developmental Disorders, 46, 3688-3699. https://doi.org/10.1007/s10803-016-2908-0

Kaartinen, M., Puura, K., Mäkelä, T., Rannisto, M., Lemponen, R., Helminen, M., Salmelin, R., Himanen, S. y Hietanen, J. (2012). Autonomic
Arousal to Direct Gaze Correlates with Social Impairments Among Children with ASD. Journal of Autism and Developmental Disorders 42, 19171927. https://doi.org/10.1007/s10803-011-1435-2

Kasari, C., Sigman, M., Mundy, P. y Yirmiya, N. (1990). Affective Sharing in the Context of Joint Attention Interactions of Normal, Autistic, and Mentally Retarded Children. Journal of Autism and Developmental Disorders, 20(1), 87-100. https://doi.org/10.1007/bf02206859

Kylliäinen, A. y Hietanen, J. K. (2006). Skin Conductance Responses to Another Person's Gaze in Children with Autism. Journal of Autism Developmental Disorders, 36(4), 517-525. https://doi.org/10.1007/s10803-006-0091-4

Kylliäinen, A., Wallace, S., Coutanche, M. N., Leppanen, J.M., Cusack, J., Bailey, A. J. y Hietanen, J. K. (2012). Affective-motivational Brain Responses to Direct Gaze in Children with Autism Spectrum Disorder. Journal of Child Psychology and Psychiatry, 53, 790-797. https://doi.org/10.1111/j.1469-7610.2011.02522.x

Lang, P. J., Greenwald, M. K., Bradley, M. M. y Hamm, A. O. (1993). Looking at Pictures: Affective, Facial, Visceral, and Behavioral Reactions. Psychophysiology, 30(3), 261-273. https://doi.org/10.1111/j.1469-

8986.1993.tb03352.x

Lang, P. J., Bradley, M. M. y Cuthbert, B. N. (1995). International Affective Picture System (IAPS): Technical Manual and Affective Ratings. Gainesville: University of Florida, Center for Research in Psychophysiology.

Lawrie, L., Jackson, M. C. y Phillips, L. H. (2018). Effects of Induced Sad Mood on Facial Emotion Perception in Young and Older Adults. Aging Neuropsychology and Cognition, 26, 319-335. https://doi.org/10.1080/13825585.2018.1438584 
Louwerse, A., Tulen, J. H., van der Geest, J. N., van der Ende, J., Verhulst, F. C. y Greaves-Lord, K. (2014). Autonomic Responses to Social and Nonsocial Pictures in Adolescents with Autism Spectrum Disorder. Autism Research, 7, 17-27. https://doi.org/10.1002/aur.1327

Machinskaya, R. I., Rozovskaya, R. I., Kurgansky, A. V. y Pechenkova E. V. (2016). Cortical Functional Connectivity during the Retention of Affective Pictures in Working Memory: EEG source Theta Coherence Analysis. Human Physiology, 42(3), 279-293.

https://doi.org/10.1134/S0362119716020122

Mathersul, D., McDonald, S. y Rushby, J. (2013a). Automatic Facial Responses to Affective Stimuli in High-functioning Adults with Autism Spectrum Disorder. Physiology \& Behaviour 109, 14-22. https://doi.org/10.1016/j.physbeh.2012.10.008

Mathersul, D., McDonald, S. y Rushby, J. (2013b). Automatic Facial Responses to Briefly Presented Emotional Stimuli in Autism Spectrum Disorders. Biological Psychology, 94, 397-407. https://doi.org/10.1016/j.biopsycho.2013.08.004

Niedenthal, P., Halberstadt, J. y Margolin, J. (2000). Emotional State and the Detection of Change in Facial Expression of Emotion. European Journal of Social Psychology, 30(2), 211-222. https://doi.org/10.1002/(SICI)10990992(200003/04)30:2\%3C211::AIDEJSP988\%3E3.0.CO;2-3

Nielen, M. M., Heslenfeld, D. J., Heinen, K., Van Strien, J. W., Witter, M. P., Jonker, C. y Veltman, D. (2009). Distinct Brain Systems Underlie the Processing of Valence and Arousal of Affective Pictures. Brain Cognition, 71, 387-396. https://doi.org/10.1016/j.bandc.2009.05.007

Ovsyannikova, V. (2016). The Effects of Mood on Emotion Recognition and its Relationship with the Global vs. Local Information Processing Styles.
Higher School of Economics Research Paper No. WP BRP 60/PSY/2016.

Pelphrey, K. A., Sasson, N. J., Reznick, J. S., Paul, G., Goldman, B. D. y Piven, J. (2002). Visual Scanning of Faces in Autism. Journal of Autism and Developmental Disorders, 32(4), 249-261. https://doi.org/10.1023/a:1016374617369

Peterman, J. S. y Park, S. (2017). Subtle Cues Missed: Impaired Perception of Emotion from Gait in Relation to Schizotypy and Autism Spectrum Traits. Schizophrenia Research, 183, 157-160. https://doi.org/10.1016/j.schres.2016.11.003

Powell, M. y Hemsley, D. R. (1984). Depression: A Breakdown of Perceptual Defence? The British Journal of Psychiatry, 145, 358-362. https://doi.org/10.1192/bjp.145.4.358

Redondo, J. y Fernández-Rey, J. (2010). Reconocimiento de fotografías de contenido emocional: Efectos de la valencia cuando se controla el arousal [Recognition Memory for Emotional Pictures: Effects of the Valence when the Arousal is Controlled]. Psicológica, 31(1), 6586.

Riby, D. M., Doherty-Sneddon, G. y Whittle, L. (2012). Face-to-face Interference in Typical and Atypical Development. Developmental science, 15(2), 281$291 . \quad$ https://doi.org/10.1111\%2Fj.14677687.2011.01125.x

Rojahn, J., Esbensen, A. J. y Hoch, T. A. (2006). Relationships between Facial Discrimination and Social Adjustment in Mental Retardation. American Journal of Mental Retardation, 111, 366-377. https://doi.org/10.1352/08958017(2006)111[366:RBFDAS]2.0.CO;2

Rottenberg, J., Joormann, J., Brozovich, F. y Gotlib, I. H. (2005). Emotional Intensity of Idiographic Sad Memories in Depression Predicts Symptom Levels 1 Year Later. Emotion, 5(2), 238-242. https://doi.org/10.1037/1528-3542.5.2.238 
Schechtman Belham, F., Tavares, M. C., Satler, C., Garcia, A., Rodrigues, R. C., de Sá Canabarro, S. y Tomaz, C. (2017). Negative Facial Expressions But Not Visual Scenes - Enhance Human Working Memory in Younger and Older Participants. Frontiers in Pharmacology, 8, 668. https://doi.org/10.3389\%2Ffphar.2017.00668

Schmid, P. C. y Mast, M. S. (2010). Mood Effects on Emotion Recognition. Motivation and Emotion, 34(3), 288-292. https://doi.org/10.1007/s11031$010-9170-0$

Sparrow, S., Balla, D. y Cicchetti, D. (1984). The Vineland Adaptive Behavior Scales (Survey Form). Circle Pines, MN: American Guidance Service.

Stagg, S. D., Davis, R. y Heaton, P. (2013). Associations Between Language Development and Skin. Conductance Responses to Faces and Eye Gaze in Children with Autism Spectrum Disorder. Journal of Autism and Developmental Disorders, 43, 23032311. https://doi.org/10.1007/s10803-013-1780-4

Stagg, S. D., Slavny, R., Hand, C., Cardoso, A. y Smith, P. (2014). Does Facial Expressivity Count? How Typically Developing Children Respond Initially to Children with Autism. Autism, 18(6), 704-711. https://doi.org/10.1177\%2F1362361313492392

Surguladze, S. A., Young, A. W., Senior, C,. Brébion, G,. Travis, M. J. y Phillips, M. L. (2004). Recognition Accuracy and Response Bias to Happy and Sad Facial Expressions in Patients with Major Depression. Neuropsychology, 18(2), 212-218. https://doi.org/10.1037/0894-4105.18.2.212

Sparrow, S., Balla, D. y Cicchetti, D. (1984). The Vineland Adaptive Behavior Scales (Survey Form). Circle Pines, MN: American Guidance Service.
Uljarevic, M. y Hamilton A. (2013). Recognition of Emotions in Autism: A Formal Meta-Analysis. Journal of Autism and Developmental Disorders, 43(7), 1517-1526. https://doi.org/10.1007/s10803$012-1695-5$

Walsh, J. A., Creighton, S. E. y Rutherford, M. D. (2016). Emotion Perception or Social Cognitive Complexity: What Drives Face Processing Deficits in Autism Spectrum Disorder? Journal of Autism and Developmental Disorders, 46, 615-623. https://doi.org/10.1007/s10803-015-2606-3

Weeks S. J. y Hobson, R. P. J. (1987). The Salience of Facial Expression for Autistic Children. Journal of Child Psychology and Psychiatry, 28(1), 137-151. https://doi.org/10.1111/j.1469-

7610.1987.tb00658.x

Yi, C. Y., Murry, M. W. E. y Gentzler, A. L. (2016). Perception of Emotional Expressions in Adults: The Role of Temperament and Mood. Journal of Individual Differences, 37(1), 16-23.

Yirmiya, N., Kasari, C., Sigman, M. y Mundy, P. (1989). Facial Expressions of Affect in Autistic, Mentally Retarded and Normal Children. Journal of Child Psychology and Psychiatry, 30(5), 725-735. https://doi.org/10.1111/j.14697610.1989.tb00785.x

Zangróniz, R., Martínez-Rodrigo, A., Pastor, J. M., López, M. T. y Fernández-Caballero, A. (2017). Electrodermal Activity Sensor for Classification of Calm/Distress Condition. Sensors, 17, 2324. https://doi.org/10.3390/s17102324 


\title{
IMPACT OF EMOTIONAL ACTIVATION ON THE RECOGNITION OF EMOTIONS IN ADULTS WITH AUTISM SPECTRUM DISORDERS AND INTELLECTUAL DISABILITY
}

\author{
MARINA JODRA $^{1}$ Y DOMINGO GARCÍA-VILLAMISAR $^{1}$
}

\section{EXTENDED SUMMARY}

\section{Introduction}

One of the core symptoms of Autism Spectrum Disorders (ASD) is the deficit in the socio-emotional area. Several studies demonstrated the existence of a specific deficit in the recognition and understanding of emotions in people with autism (Blain et al., 2017; GarcíaVillamisar \& Polaino-Lorente, 2000; García-Villamisar et al., 2010 ; Uljarevic \& Hamilton, 2013). Some research limits the deficit to the recognition of very specific emotions such as fear (De Jong et al., 2008; Pelphrey et al., 2002), sadness (Boraston et al., 2007), the emotions of "positive valence" (Herpers et al., 2019) or "negative" emotions (Ashwin et al., 2006; Humphreys et al., 2007; Ioannou et al., 2017) generally showing less attention to them (Baron-Cohen et al., 2000). This deficit in the recognition of emotions is accompanied by a certain lack of interest of people with autism in the emotions of others (Begeer et al., 2006; Pelphrey et al., 2002; Weeks \& Hobson, 1987), and less attention to social stimuli (Chevallier et al., 2013; Mathersul et al., 2013a). Other research has observed a different pattern of attention to emotional stimuli, proving in people with ASD greater attention to threatening stimuli (Ghosn et al., 2019). In addition, people with ASD seem less expressive in social interactions, being more neutral than people with intellectual disabilities and typical development without ASD (Czapinski \& Bryson, 2003; Kasari et al., 1990; Stagg et al., 2014; Yirmiya et al., 1989). In people with intellectual disabilities and autism, less activation was detected in the direction of another person's gaze
(Kylliäinen \& Hietanen, 2006) or in situations of danger (Corona et al., 1998). Some research highlights the existence of significant difficulties when emotional perception is done through faces (Walsh et al., 2016; Harms et al., 2010).

One of the theories that has tried to explain these atypical response patterns in ASD, argues that having to deal with social stimuli people with autism respond with different levels of activation or arousal (Kaartinen et al., 2016; Kylliäinen et al., 2012; Riby et al., 2012). The hyperexcitation model suggests that the autonomic nervous system of individuals with ASD is "elevated", they are constantly on high alert (Bal et al., 2010; Hirstein et al., 2001). On the contrary, the hypoexcitation model, defends that people with ASD experience less excitement or reward when they attend to social stimuli (Mathersul et al., 2013b).

Some investigations show a greater tendency of subjects with negative humor due to the identification of stimuli as negative (Bouhuys et al., 1995; Niedenthal et al., 2000; Schmidt \& Mast, 2010; Yi et al., 2016) and a greater performance in this type of tasks in people with positive humor (Ovsyannikova, 2016). They have also related these variables with age, proving a greater predisposition to perceive negative stimuli when negative emotions are induced and the subject is older (Lawrie et al., 2018). On the other hand, the few studies that exist on emotional permeability in people with ASD show atypical patterns of physiological response to the induction of emotions.

In the present study, the impact of "negative" emotional induction is analyzed in a group of adults with 
ASD and intellectual disability (ID), by images of the International Affective Picture System (IAPS, Lang et al., 1995). The recognition of emotions is evaluated by the face through Facial Discrimination Battery (FDB; Rojahn et al., 2006).

\section{Method}

\section{Participants}

The sample selected to carry out this research consists of 31 adults with ASD and ID, of whom 19 are male $(61.2 \%)$ and 12 are female (38.7\%). All of them have been diagnosed by specialists in the evaluation of Autism Spectrum Disorders, according to the diagnostic criteria of the American Psychiatric Association (DSM-IV; APA, 1994).

\section{Instruments}

\section{Dependent variable: recognition of emotions through the face}

The Facial Discrimination Battery (FDB; Rojahn et al., 2006; García-Villamisar et al., 2010) evaluates facial processing using four emotional tests ("facial recognition task", "identification task", "match with the sample" and "facial discrimination task"), and two non-emotional tests ("facial discrimination task" and "match with the sample"). The percentage of correct scores are recorded for each subtest.

\section{Independent variable: induction of negative emotions}

The International Affective Picture System (IAPS; Land et al., 1995) is a standardized set of approximately 900 color slides with different emotional categories, capable of provoking emotions. It belongs to a collection of emotional stimuli, along with the IADS (International Affective Digitized Sound System) and the ANEW (Affective Lexicon of English Words), developed by the
NIMH Center for Emotion and Attention (CSEA) of the University of Florida.

The IAPS measures the following emotional dimensions: pleasant / unpleasant; calm / excitement (arousal) and uncontrolled / controlled.

\section{Procedure}

Once the participants were selected and after having obtained informed consent from all the legal tutors, the evaluation process began. All tests were applied by a single examiner, individually and in quiet rooms.

For the selection of images of the IAPS in this study, the table of means and standard deviations of each image in the general population has been consulted in the manual (Lang et al., 2005). For the classification of the images, the authors of the IAPS were consulted, proposing their own method of selection.

The objective has been to see if there is displeasure, excitement and lack of control. For this, the participants were randomly divided into two subgroups, one for induction and one for non-induction. To both groups the Rojahn Emotional Recognition Battery was applied before the experimental induction. After viewing the images of the IAPS, the two groups performed again the tasks of the Rojahn Battery of Emotions.

\section{Results}

An ANOVA of repeated measures was performed and the results demonstrated the existence of a certain emotional "impermeability", since the induction of emotions did not influence the subsequent emotional recognition $(\mathrm{F}(7,15)=1.42, \quad p=.26, \quad \eta 2=.39)$. The measurement time, first or second application of tasks, significantly influences emotional performance $(\mathrm{F}(7.15)=3.60, p=.01, \mathrm{\eta} 2=.62)$. 


\section{Discussion}

These results are consistent with the hypoexcitation model, which argues that people with ASD experience less excitement or reward when they process social stimuli.

These findings are contrary to what is observed in a population without ASD, since when viewing negative valence images the subsequent recognition of emotions is modified (Daudelin-Peltier et al., 2017; Gordillo et al., 2010; Redondo \& Fernández-Rey, 2010; Rozovskayaa et al., 2016; Schechtman Belham et al., 2017). One more difference is found regarding to the adult population without ASD, since a greater predisposition has been detected to perceive negative stimuli when negative emotions are induced and the person is older (Lawrie et al., 2018 ).

The previous literature, that is consistent with these findings, are those studies about the ability to respond to the emotions of people with autism. Among these investigations are those that defend the existence of a specific deficit in the recognition and understanding of emotions (Ashwin et al., 2006; Blain et al., 2017; Boraston et al., 2007; De Jong et al., 2008; García-Villamisar \& Polaino-Lorente, 2000; García-Villamisar et al., 2010; Humphreys et al., 2007; Ioannou et al., 2017; Pelphrey et al., 2002; Uljarevic \& Hamilton, 2013). This deficit is accompanied by some lack of interest on the part of people with autism in the emotions of others (Begeer et al., 2006; Pelphrey et al., 2002; Weeks \& Hobson, 1987), and less attention to social stimuli (Chevallier et al., 2013; Mathersul et al., 2013a). In addition, people with ASD seem less expressive in social interactions, being more neutral than people with intellectual disabilities and typical development without ASD (Czapinski \& Bryson, 2003; Kasari et al., 1990; Stagg et al., 2014; Yirmiya et al., 1989).

The responses tend to be less empathic and fewer heart rate have been detected in people with ASD in response to other people's emotions, comparing them with people with typical development (Corona et al., 1998). In people with intellectual disabilities and autism less excitement or attention was detected in the direction of another person's gaze (Kylliäinem \& Hietanen, 2006) or in situations of danger (Corona et al., 1998). An altered physiological reaction to emotional induction with the IAPS has been observed, through blood pressure and heart rate (Bölte et al., 2008; Kaartinen et al., 2012; Kylliäinen et al., 2012; Mathersul et al., 2012), and significant correlations between the galvanic response to social stimuli and the delay in language development (Stagg et al., 2013). These findings can also be analyzed from this possible emotional "impermeability" that would produce these atypical patterns of physiological responses.

Finally, these results show a certain specificity in the perception of emotional stimuli in people with ASD. An emotional "impermeability" is observed, which should be taken into account when developing therapeutic interventions adapted to these characteristic. 
118 ACCIÓN PSICOLÓGICA, diciembre 2019, vol. 16, n'. 2, 103-118. ISSN: 2255-1271 https://doi.org/10.5944/ap.16.2.25566 